\title{
25 Research Soure \\ MiR-130a-3p Regulates the Glycolysis via Targeting \\ PDK1 in Hepatocellular Carcinoma
}

\section{Hai-Long Li}

Harbin Medical University

Jie Shi

Harbin Medical University

Qi Qi

Harbin Medical University

Yue Huang

Harbin Medical University

Chi Liu

Harbin Medical University

Ping Lin

Harbin Medical University

Jie-Bing Tang

Harbin Medical University Cancer Hospital

Xiaoguang Yu ( $\nabla$ yuxg@hrbmu.edu.cn )

Harbin Medical University https://orcid.org/0000-0002-6293-4285

\section{Research Article}

Keywords: PDK1, HCC, glycolysis, miR-130a-3p

Posted Date: December 20th, 2021

DOI: https://doi.org/10.21203/rs.3.rs-1155969/v1

License: (c) (1) This work is licensed under a Creative Commons Attribution 4.0 International License.

Read Full License 


\section{Abstract}

MiR-130a-3p has been certified to have low expression in several types of tumors. However, the function of miR-130a-3p in glucose metabolism and hepatocellular carcinoma progression is still elusive. Here we report that miR-130a-3p has explicitly low expression in human HCC tissues and cells and is closely related to the patient's tumor size and grade. Overexpression of miR-130a-3p significantly inhibits the glucose metabolism, proliferation and migration of HCC cells in vitro. In order to further study the effects of miR-130a-3p in the glucose metabolism of HCC cells, we found that overexpression of miR-130a-3p significantly inhibited the expression of pyruvate dehydrogenase kinase 1 (PDK1). Consistently, we confirmed that PDK1 is the target gene of miR-130a-3p through dual luciferase reporter gene assays. Cell rescue experiments showed that PDK1 inhibitors reversed the enhancement of cell proliferation, migration and glucose metabolism by miR-130a-3p inhibitor in Hep3B cells. In terms of mechanism, overexpression of miR-130a-3p targeted and inhibited the expression of PDK1, after which pyruvate dehydrogenase $(\mathrm{PDH})$ is activated, thus glycolysis is inhibited, the production of lactic acid and ATP is reduced, and the ability to proliferate and migrate in HCC cells is weakened. In conclusion, our study highlights efforts to target PDK1 and miR-130a-3p as potential therapeutic strategies for the treatment of HCC.

\section{Introduction}

There are approximately 854,000 new cases of liver cancer each year, of which $85 \%-90 \%$ are hepatocellular carcinoma, making it the sixth most common cancer in the world ${ }^{1,2}$. According to the American Cancer Society, the incidence of liver cancer is growing at the fastest rate among all cancers in the United States. In the past ten years, the number of HCC cases has doubled, possibly due to obesity/diabetes ${ }^{3}$. Due to lack of treatment, $\mathrm{HCC}$ is the second leading cause of cancer deaths in adult men ${ }^{4,5}$. Thus, new therapeutic approaches for $\mathrm{HCC}$ are urgently needed.

In the past two decades, microRNAs (miRNAs) have been identified by researchers as non-coding components of the human genome and are key mediators of organ development and human diseases ${ }^{6}$. MiRNAs are a class of small noncoding RNA that act as endogenous regulators of gene expression ${ }^{7}$. Accumulated evidence indicated that miRNAs play essential role in regulating various cell activities, such as cell growth, differentiation, apoptosis, and tumorigenesis ${ }^{8,9}$. For example, studies have shown that miR-30a and miR-26a inhibit hepatocellular carcinoma tumor metastasis ${ }^{10,11}$; miR-203 inhibits colorectal cancer cell migration and invasion ${ }^{12}$; the expression of miR-7, miR-153, miR-21, miR-34a, miR-128, etc. in a variety of tumor tissues has undergone significant changes and participates in the occurrence and development of tumors ${ }^{13-16}$. As a significant tumor inhibitor, miR-130a-3p has been proved to be in low expression in several kinds of cancers and was supposed to be the candidate for the cancer diagnosis as a promising biomarker ${ }^{17-19}$. Studies have reported that miR-130a-3p inhibits migration and invasion by regulating $\mathrm{RAB} 5 \mathrm{~B}$ in human breast cancer stem cell-like cells ${ }^{20}$, miR-130a-3p inhibits the viability, proliferation, invasion, and cell cycle, and promotes apoptosis of nasopharyngeal carcinoma cells by suppressing $\mathrm{BACH} 2$ expression ${ }^{21}$, and miR-130a-3p regulates cell migration and invasion via inhibiting 
Smad4 in gemcitabine resistant hepatoma cells ${ }^{22}$. On the metabolic side, it has recently been reported that LncRNA HOTAIR knockdown inhibits glycolysis by regulating miR-130a-3p/HIF1A in hepatocellular carcinoma under hypoxia ${ }^{23}$, manifesting the possible involvement of miR-130a-3p in the regulation of glucose metabolism.

To date, it has been demonstrated that one of the important hallmarks of cancer biology is metabolic reprogramming, of which the most important feature is aerobic glycolysis, and that is, there exists the significant increase in glycolytic activity even in the presence of abundant oxygen which has been discovered by Otto Warburg nighty years ago, also known as "Warburg effect" ${ }^{24}$. While the Warburg effect is now well appreciated, the mechanisms driving aerobic glycolysis are not fully understood ${ }^{25}$. This metabolic shift confers a biological advantage to cancer cells, thus supporting both initiation and progression of tumors ${ }^{26}$. Understanding the underlying mechanisms of metabolic alterations in cancer has the potential to improve cancer diagnosis and provide the foundation for novel therapeutic approaches. PDK plays an important role in glucose metabolism. PDK is a 45 48kda serine-specific kinase, including PDK1-4, which is the main cause and participant of phosphorylation and inactivation of pyruvate dehydrogenase E1a. PDK regulates the activity of the pyruvate dehydrogenase complex (PDC), which catalyzes the conversion of pyruvate to acetyl-CoA. Recent studies have shown that PDKs are generally overexpressed in a variety of tumor cells, such as multiple myeloma ${ }^{27}$, hepatocellular carcinoma $^{28}$ and malignant glioma ${ }^{29}$. As an essential glycolytic enzyme, PDK1 is closely related to tumor proliferation, metastasis and poor prognosis ${ }^{30,31}$.

In this study, we report that miR-130a-3p is low expressed in HCC clinical tissue specimens and cells and that is related to the patient's tumor size and grade. We discovered for the first time that miR-130a-3p inhibits the proliferation and migration of HCC cells by inhibiting the aerobic glycolysis of HCC cells. Also for the first time, it was discovered and confirmed that PDK1, as a new target molecule of miR-130a-3p, participates in the process of affecting glucose metabolism reprogramming of HCC. These results suggest that a novel miR-130a-3p/PDK1 axis may act as a potentially therapeutic target and prognostic biomarker for human HCC.

\section{Materials And Methods}

\subsection{Clinical tissue chips and Cell culture}

30 cases of HCC and adjacent tissue chips were purchased from Shanghai Auto Biotech Co., Ltd. All specimens were confirmed by pathological diagnosis and the ethics committee. HCC cell lines (HepG2, Huh7 and Hep3B), normal human hepatic cell line LO2 and 293T cells were purchased from the Cell Bank of Chinese Academy of Sciences (Shanghai, China). All cells were cultured at $37^{\circ} \mathrm{C}$ under $5 \% \mathrm{CO} 2$ in highglucose Dulbecco's modified Eagle medium (DMEM) medium (meilunbio, Dalian), supplemented with 10\% FBS (Gibco, New York), 1\% antibiotics (penicillin and streptomycin; $100 \mathrm{U} / \mathrm{ml}$ ) (Gibco).

\subsection{MiRNA mimics, inhibitors and OSU-03012}


MiRNA mimics, inhibitors and corresponding NC were purchased from Ruibo Company (Shanghai, China). An amount of 50nM miRNA mimics or 100nM inhibitors were transfected into Hepg2 or Hep3B cells in 60-mm dishes with Lipofectamine 3000 (Invitrogen) according to the manufacturer's instruction. OSU-03012 was purchased from Beyotime. According to the instructions, the cells were cultured for 24-hr, and then OSU-03012 $(5 \mu \mathrm{M})$ dissolved in DMSO(final concentration $\leq 0.1 \%$ ) was added to DMEM medium containing $1 \%$ serum at different time intervals.

\subsection{RNA extraction and qRT-PCR}

Total RNA was extracted from the cells by Trizol RNA isolation reagent (Takara, Beijing, China) according to the manufacturer's protocol, which then was reversed transcribed into cDNA by the PrimeScript RT reagent Kit with gDNA Eraser (Takara). Real-time quantitative PCR was performed by the SYBR select supermix kit (Thermo Fisher Scientific, Shanghai, China) in an ABI Prism 7500 PCR instrument. Relative gene expression levels were calculated by the 2- $\Delta \Delta$ ct method with GAPDH or U6 as the internal control. The PCR primers were designed and synthesized by GenePharma (Shanghai, China) (Table 1). 
Table 1

Primer sequence

\begin{tabular}{|c|c|}
\hline Gene & Primer sequence \\
\hline \multirow[t]{2}{*}{ miR-130a-3p } & F: 5'- TTGCGATTCTGTTTTGTGCT-3' \\
\hline & R: 5'-GTGGGGTCCTCAGTGGG -3' \\
\hline \multirow[t]{2}{*}{ U6 } & F: 5'-GCAAATTCGTGAAGCGTTCC -3' \\
\hline & R: 5'-AACGCTTCACGAATTGCGT -3' \\
\hline \multirow[t]{2}{*}{ PDK1 } & F: 5'- CTGTGATACGGATCAGAAACCG -3' \\
\hline & R:5'- TCCACCAAACAATAAAGAGTGCT-3' \\
\hline \multirow[t]{2}{*}{ PDK2 } & F: 5'-ATGAAAGAGATCAACCTGCTTCC -3' \\
\hline & R:5'- GGCTCTGGACATACCAGCTC -3' \\
\hline \multirow[t]{2}{*}{ PDK3 } & F: 5'- AATGGCACAAGGAGTGATTGAA -3' \\
\hline & R:5'- CATCCGCCACGTTACAGGT -3' \\
\hline \multirow[t]{2}{*}{ PDK4 } & F: 5'- GGAAGCATTGATCCTAACTGTGA-3' \\
\hline & R:5'-GGTGAGAAGGAACATACACGATG-3' \\
\hline \multirow[t]{2}{*}{ EN01 } & F: 5'- TGAGGGAATGAGTGACGGC -3' \\
\hline & R:5'- ACAGCCTTTGAGACACCCTTC-3' \\
\hline \multirow[t]{2}{*}{ GLUT1 } & F: 5'- ATCAACCGCAACGAGGAGAAC -3' \\
\hline & R: 5'- CACCACAAACAGCGACACGAC -3' \\
\hline \multirow[t]{2}{*}{ GLUT2 } & F: 5'- AAGCCCCAGATACCTTTACAT -3' \\
\hline & R: 5'- AACAAACATCCCACTCATTCC -3' \\
\hline \multirow[t]{2}{*}{ GLUT3 } & F: 5'- TCAGGCTCCACCCTTTGCGGA -3' \\
\hline & R: 5'- TGGGGTGACCTTCTGTGTCCCC-3' \\
\hline \multirow[t]{2}{*}{ G6PD } & F: 5'- ACTCCCGAAGAGGGGTTCAAGG -3' \\
\hline & R:5'-CCAGCCTCCCAGGAGAGAGGAAG-3' \\
\hline \multirow[t]{2}{*}{ PDHB } & F:5'-GGAGGATCCATGTCTGATAAAAAAATA-3' \\
\hline & R:5'-TTACTCGAGCTAAAATTTAAATGAAGT-3' \\
\hline \multirow[t]{2}{*}{ HK2 } & F: 5'- TTGACCAGGAGATTGACATGGG -3' \\
\hline & R: 5'- CAACCGCATCAGGACCTCA -3' \\
\hline
\end{tabular}




\begin{tabular}{|ll|}
\hline Gene & Primer sequence \\
\hline PKM2 & F: 5'-ATAACGCCTACATGGAAAAGTGT -3' \\
& R: 5'- TAAGCCCATCATCCACGTAGA -3' \\
\hline LDHA & F: 5'- ATGGCAACTCTAAAGGATCAGC -3' \\
& R: 5'- CCAACCCCAACAACTGTAATCT -3' \\
PGK1 & F: 5'- TGGACGTTAAAGGGAAGCGG -3' \\
& R:5'- GCTCATAAGGACTACCGACTTGG -3' \\
GAPDH & F: 5'-GGACCTGACCTGCCGTCTAG -3' \\
& R: 5'-GTAGCCCAGGATGCCCTTGA -3' \\
\hline
\end{tabular}

\subsection{Western blotting}

Cell lysis was performed by RIPA buffer (Beyotime) added with proteinase inhibitor (Sigma-Aldrich) on ice and centrifuged for 15 mins at $4^{\circ} \mathrm{C}$ at $12000 \mathrm{~g}$. Protein concentrations were determined by a BCA assay kit (Beyotime). Protein samples in equal quality were isolated by $10 \%$ SDS-PAGE and then transferred to NC membranes (EMD Millipore), which were blocked in QuickBlock Blocking Buffer (Beyotime) for 10-15 mins. The membranes were then incubated with monoclonal rabbit primary anti-bodies against PDK1, Ecadherin, Vimentin, CyclinD1, PCNA (Abcam, Cambridge, UK) or GAPDH (Cell Signaling Technology, Danvers, MA) at $4^{\circ} \mathrm{C}$ overnight. Subsequently, the membranes were incubated with horseradish peroxidase-linked secondary antibodies for 2-hr at room temperature. The blots were visualized by the substrate BeyoECL Plus (Beyotime Institute of Biotechnology) and imaged by a Tanon 5200 Chemiluminescent Imaging system.

\subsection{Cell transfection}

Before transfection, HepG2 and Hep3B cells were seeded in small dishes for 24-hr. The synthesized miR130a-3p mimics/inhibitors or corresponding NC was transfected via Opti-MEM and Lipofectamine 3000 Ttansfection Kit (Thermo Fisher Scientific, Shanghai, China). After 48-hr, the expression of miR-130a-3p could be observed.

\subsection{Luciferase assays}

Targeting relationship and binding sites between miR-130a-3p and PDK1 were predicted at ENCORI (http://starbase.sysu.edu.cn/). Two psiCHECK2 plasmids, one containing the wild type (WT) PDK1 3'untranslated region (3'-UTR) sequence (WT_PDK1) and the other containing mutant (MUT) PDK1 3'-UTR sequence (MUT_PDK1, with miR-130a-3p binding site voided), were manufactured by GenePharma (Sangon Biotech, Shanghai, China). The WT_PDK1 or MUT_PDK1 plasmids were co-transfected with miR- 
130a-3p mimic or mimic NC respectively into 293T cells. The luciferase activity was detected via luciferase assay kit (Beyotime, Shanghai, China).

\subsection{Cell counting kit-8 (CCK-8) assay}

After 48-hr transfection, the cells of each group were detached with trypsin, makeing the cell suspension well dispersed, and the cells were counted and diluted to a final concentration seeded in a 96-well plate at about $200 \mu \mathrm{l}$ cell suspension/well $(1 \times 103$ cells). Five replicate wells (0-hr gruop, 24-hr group, 48-hr group, 72-hr group and blank control) were set, and then 10 $\mu$ l CCK-8 solution (Sigma, St. Louis, MO) was added for 2-hr incubation at each group. The OD value of each well was measured at a wavelength of $450 \mathrm{~nm}$ by the microplate reader, and the cell viability curve was plotted after obtaining the average OD value at four time points.

\subsection{Colony formation assay}

After 48-hr transfection, the cells of each group were detached with trypsin, the cell suspension was well dispersed, and about $1.0 \times 103$ cells were inoculated in culture medium dishes containing 4 mL medium and cultured for $2-3$ weeks at $37^{\circ} \mathrm{C}$ with $5 \% \mathrm{CO} 2$.When cell masses were visible to the naked eyes, cells masses were fixed in formaldehyde and stained by Crystal Violet Staining Solution. With above procedures accomplished, photograph colony formation dishes and count cell masses more than 50 cells under the microscope.

\subsection{Transwell assay}

Cell migration was measured by Transwell migration assay. After 48-hr transfection, the cells of each group were detached with trypsin. Approximate $2.5 \times 104$ cells and $200 \mu$ l of dual-free medium were added to the Transwell chambers and $600 \mu \mathrm{l}$ of complete medium were added to the 24-well plate. Next, the chamber was cultured at $37^{\circ} \mathrm{C}$ for $24-\mathrm{hr}$ in incubator with $5 \% \mathrm{CO} 2$. Afterward, rinsed via $1 \times$ PBS, cells in the chambers were fixed by formaldehyde 5 mins and stained by crystal violet. After that, the unmigrated cells in outer membrane of chambers were wiped off by cotton swabs and the migrated cells in inner membrane of chambers were observed, photographed under the microscope and counted.

\subsection{Wound healing assay}

After transfection, cells were cultured in small dishes and incubated overnight to reach $80 \%$ distribution. The cells evenly bespreading were made wound areas by the 200ul pipette tip and cultured with DMEM medium without FBS at $37^{\circ} \mathrm{C}$ with $5 \% \mathrm{CO} 2$. The process of cells migration into the wound area was imaged at 0-hr, 24-hr and 48-hr respectively. The wound widths were measured and the migration ability was analyzed.

\subsection{Measurement of lactate production}

After transfection, cells were cultured in the medium and then supernatants were respectively collected for further analysis. The lactate production level was measured by lactate assay kit (Jian Cheng 
Bioengineering Institute, Nanjing, China) according to the manufacturer's instruction. The numerical values of results are normalized by protein concentration.

\subsection{Measurement of ATP production}

Intracellular ATP levels were measured by a bioluminescence method with the ATP Assay Kit (Jian Cheng Bioengineering Institute, Nanjing, China) according to the manufacturer's instructions. Luminescence was measured by a luminescence reader (Promega) and the numerical values of results are normalized by protein concentration.

\subsection{Measurement of PDH activity}

According to the manufacturer's instructions, cell lysates and PDH activity assay kit (Solarbio) was utilized to measure the Intracellular PDH activity.

\subsection{Statistical analysis}

All quantitative experiments were repeated at least three times. The measurement data were demonstrated as mean \pm SD. Student's t test or one-way ANOVA was applied to determine statistical significance. All experiments were performed at least 3 biological replicates. Statistical significance is displayed as $* p<.05$.

\section{Results}

\subsection{MiR-130a-3p was down expressed in HCC tissues and cell lines}

Firstly, we used the ENCORI (http://starbase.sysu.edu.cn/) database to predict the expression of miR130a-3p, we observed that the expression level of miR-130a-3p was decreased in HCC (Figure 1a). And we tested the expression level of miR-130a-3p in 30 cases of HCC and adjacent tissue chips. The results showed that miR-130a-3p had a low expression in 25 HCC tissues (83.3\%), a high expression in 5 cases of HCC tissues (16.7\%)(Figure 1b). Further In order to analyze the correlation of differentiated expression of miR-130a-3p between HCC and adjacent tissues as well as the clinicopathological characteristics, we drew the ROC curve of miR-130a-3p, and the area under the ROC curve was 0.641 (Figure 1c), indicating that miR-130a-3p can be deemed as a potentially independent marker for the prognosis of HCC. We further detected the expression of miR-130a-3p in HCC cells via qRT-PCR. The results showed that miR130a-3p is low expression in HCC cells (Figure 1d). 
According to the relationship between the expression of miR-130a-3p in HCC tissues and the median ratio, we divided $30 \mathrm{HCC}$ patients into two groups: miR-130a-3p high expression group $(n=15$, expression rate $\geq$ median ratio) and miR-130a-3p low expression group ( $n=15$, expression rate $\leq$ median ratio). The results show that the expression of miR-130a-3p is related to the size of the patient's tumor and TNM stage while has nothing to do with the patient's gender, age, and tumor location (Table 2). The results show that miR-130a-3p is low expressed in HCC and closely related to the occurrence and development of HCC.

Table 2

The correlation between miR-130a-3p expression and HCC patients' clinicopathological factors $\left(N=30\right.$, ${ }^{*} p$ $<0.05)$

\begin{tabular}{|c|c|c|c|c|}
\hline $\begin{array}{l}\text { Clinical } \\
\text { characteristics }\end{array}$ & $\begin{array}{l}\text { Case } \\
\text { number }\end{array}$ & $\begin{array}{l}\text { Low miR-130a-3p } \\
\text { expression }\end{array}$ & $\begin{array}{l}\text { High miR-130a-3p } \\
\text { expression }\end{array}$ & Value \\
\hline Sex & & & & 0.074 \\
\hline Male & 23 & 12 & 11 & \\
\hline Female & 7 & 3 & 4 & \\
\hline Age & & & & 0.199 \\
\hline$\leq 60$ years & 19 & 10 & 9 & \\
\hline$>60$ years & 11 & 5 & 6 & \\
\hline Tumor size & & & & $0.026 *$ \\
\hline$\leq 5 \mathrm{~cm}$ & 10 & 4 & 6 & \\
\hline$>5 \mathrm{~cm}$ & 20 & 9 & 11 & \\
\hline TNM stage & & & & 0.031 * \\
\hline$\nabla / \nabla$ & 24 & 14 & 10 & \\
\hline$\nabla / \nabla$ & 6 & 2 & 4 & \\
\hline Lesion location & & & & 0.126 \\
\hline $\begin{array}{l}\text { Left lobe of the } \\
\text { liver }\end{array}$ & 9 & 6 & 3 & \\
\hline $\begin{array}{l}\text { Right lobe of the } \\
\text { liver }\end{array}$ & 21 & 10 & 11 & \\
\hline
\end{tabular}

\subsection{MiR-130a-3p inhibits HCC cells proliferation}

To investigate the effect of abnormally expressed miR-130a-3p on the function of HCC cells, we transfected miR-130a-3p mimic or corresponding NC into HepG2 cells with relatively low miR-130a-3p 
expression, and simultaneously transfected miR-130a-3p inhibitor or corresponding NC into Hep3B cells with relatively high expression of miR-130a-3p, then the expression of miR-130a-3p was detected by qRTPCR. We could note that the expression of miR-130a-3p was significantly increased in HepG2 cells and was significantly decreased in Hep3B cells compared with the control group (Figure 2a). Thus we successfully obtained HepG2 cells up-regulating miR-130a-3p and Hep3B cells down-regulating miR130a-3p. Next, we evaluated the effect of miR-130a-3p on the proliferation in HCC cells. First, we utilized the above cell lines to detect cell proliferation via CCK-8 and clone formation experiments, then Western blot is used to determinate the expression of proliferation-related proteins, CyclinD1 and PCNA. The results verified that the cell viability and colony formation rate were decreased by the miR-130a-3p mimic, while the miR-130a-3p inhibitors got the opposite results (Figure 2b, c). The expression of CyclinD1 and PCNA in HepG2 cells with miR-130a-3p up-regulated significantly reduced, while Hep3B cells with miR$130 a-3 p$ down-regulated got the opposite result (Figure $2 \mathrm{~d}$ ). The above results demonstrated that miR130a-3p inhibited the proliferation of HCC cells.

\subsection{MiR-130a-3p inhibits HCC cells migration}

To illuminate whether miR-130a-3p affects the migration ability of HCC cells, cell scratch assay and Transwell assay were used to examine the changes of cell migration after transfection. In addition, we performed Western blotting to detect expression of EMT-related proteins including E-cadherin and Vimentin. The results showed that the cell migration rate and the number of cell migration were significantly reduced by the miR-130a-3p mimic, while the miR-130a-3p inhibitors got the opposite results (Figure $3 a, b$ ). In terms of the expression of EMT-related protein, E-cadherin was significantly increased and Vimentin was significantly reduced in HepG2 cells with miR-130a-3p up-regulated, while Hep3B cells with miR-130a-3p down-regulated got the opposite result (Figure 3c). The results suggested that miR130a-3p inhibited the migration of HCC cells.

\subsection{MiR-130a-3p suppresses Warburg effect in HCC cells}

Metabolic reprogramming has been regarded as one of the important signs of cancer. Glucose metabolic reprogramming is a significant part of tumor cell metabolic reprogramming. Its characters are excessive glycolysis, changes of the expression level of enzymes related to glucose metabolism and so on. In order to study whether miR-130a-3p has an effect on the glucose metabolism of HCC cells, we firstly detected the production of lactic acid in the two HCC cell lines obtained before. The results showed that the cells that up-regulated the expression of miR-130a-3p significantly reduced lactate production, while the cells that down-regulated the expression of miR-130a-3p got the opposite result (Figure 4a). Next, we tested the ATP levels in the same obtained cell lines. Similarly, the results showed that the ATP level in the cells that up-regulated the expression of miR-130a-3p was significantly reduced, while the cells that down-regulated the expression of miR-130a-3p got the opposite result (Figure 4b). Pyruvate dehydrogenase complex (PDC) is composed of five main subunits, and pyruvate dehydrogenase (PDH) is an important component of the complex. PDKs can inactivate PDH by phosphorylating the E1a subunit of PDH and prevent the conversion of pyruvate into acetyl-CoA, which thereby makes pyruvate enter the glycolytic pathway and be converted into lactic acid. Therefore, we tested the activity of PDH in the two HCC cell lines obtained 
above. The results showed that the PDH activity in the cells that up-regulated miR-130a-3p expression was significantly increased, while the cells that down-regulated miR-130a-3p expression got the opposite result (Figure 4c). The above results indicate that the overexpression of miR-130a-3p can transform HCC cells from anaerobic glycolysis to aerobic oxidation, that is, HCC cells undergo glycometabolism reprogramming.

\subsection{PDK1 is a direct target of miR-130a-3p}

The underlying mechanism of miR-130a-3p affecting glucose metabolism of HCC cells is still dim. Therefore, we firstly used qRT-PCR to detect the expression of glucose metabolism related genes in HepG2 cells overexpressing miR-130a-3p. The results showed that miR-130a-3p could decrease the expression level of PDK1 mRNA (Figure 5a), suggesting that $P D K 1$ may be a gene targeted and regulated by miR-130a-3p. Bioinformatic analysis revealed that there is a potential miR-130a-3p binding fragment in the 3'-untranslated region (UTR) of PDK1 (Figure 5d). Next, We used ENCORI (http://starbase.sysu.edu.cn/) database to analyze the correlation between miR-130a-3p and PDK1 expression in HCC tissues, the results showed that the expression of miR-130a-3p and PDK1 was negatively correlated in HCC (Figure $5 b$ ). Western blot results showed that the expression of PDK1 protein was significantly decreased in the cells by miR-130a-3p mimic, while got the opposite result in the cells by miR-130a-3p inhibitor (Figure 5c). We then cloned that potential miR-130a-3p binding fragment as well as its mutated form into a luciferase expressing psicheck2 reporter vector (Figure $5 \mathrm{~d}$ ). The dual fluorescein enzyme reporter gene detection confirmed that miR-130a-3p reduced the luciferase activity of the WT_PDK1 reporter gene while had no significant effect on the luciferase activity of the MUT_PDK1 reporter gene (Figure 5e). These results suggested that PDK1 was a target gene of miR-130a-3p, and it was negatively regulated by miR-130a-3p.

\subsection{PDK1 mediates miR-130a-3p-induced effects on HCC cells proliferation, invasion and Warburg effect}

We first applied PDK1 inhibitor (OSU-03021) to treat Hep3B cells transfected with miR-130a-3p inhibitor or corresponding NC, and CCK-8 assay and Transwell assay were employed respectively to test the cell proliferation and migration ability changes. These results indicated that the enhancement of viability and migration ability caused by miR-130a-3p inhibitor in Hep3B cells was reversed by OSU-03012, Meanwhile, compared with the cells treated with osu-03012 alone, the cell activity and migration ability of miR-130a$3 p$ transfected cells treated with osu-03012 were enhanced(Figure $6 a, b)$. These evidences fully prove that miR-130a-3p affects the phenotype of HCC cells by regulating PDK1. Next, we sought to examine whether PDK1 was involved in decreased glycolytic metabolism regulated by miR-130a-3p. We found that when PDK1 was inhibited by OSU-03012 in Hep3B cells, the effect of miR-130a-3p inhibitor on glucose uptake and lactate production was markedly attenuated (Figure $6 \mathrm{c}, \mathrm{d}$ ). Likewise, the decrease in PDH activity was also reversed (Figure 6e), therefore, establishing PDK1 as a functional downstream target of miR$130 a-3 p$ in regulation of glycolytic metabolism.

\section{Discussion}


$\mathrm{HCC}$ is the third leading cause of cancer death in the world. Despite the improvement of treatment methods, the overall survival rate is still not optimistic due to its high metastasis rate ${ }^{32}$. Therefore, it is urgent to deeply understand the molecular mechanism of HCC progression and find new targets to block tumor progression. As a new hallmark of HCC, aerobic glycolysis has been found to be regulated by a variety of mechanisms, among which non coding RNA, especially miRNA and IncRNA, can regulate Warburg effect, mainly by regulating glycolytic enzyme expression or glycolytic related pathways $\mathrm{s}^{33}$. Therefore, it is very vital to understand the mechanism of aerobic glycolysis disorder in tumor cells for targeted tumor therapy. In this study, we confirmed that miR-130a-3p was significantly down-regulated in $\mathrm{HCC}$ tissues and cell lines. By means of the analysis of clinical data, we found that the decrease of miR$130 a-3 p$ was remarkably correlated with clinical stage and tumor size, suggesting that miR-130a-3p may be involved in the progression of HCC. In addition, the overexpression of miR-130a-3p significantly inhibited the production of lactic acid and ATP and activated the activity of pyruvate dehydrogenase (PDH) by targeting PDK1 in HepG2 cells.

Many studies have found that the phenotypes of proliferation, metastasis and angiogenesis of HCC are related to enhance aerobic glycolysis. On one hand, there is rapid ATP production during aerobic glycolysis, enabling the tumor to adapt to its microenvironment which is short of energy resources ${ }^{34}$. On the other hand, the enhanced aerobic metabolism is accompanied by activated glycolytic flux, with increased amounts of metabolic intermediates production, such as dihydroxyacetone phosphate (DHAP, which can be used for the synthesis of triglycerides and phospholipids), 3-phosphoglycerate (3-PG, which can be used for the synthesis of amino acids). Then, they can enter other metabolic pathways to synthesize nucleotides, lipids and proteins, so as to promote the proliferation and metastasis of cancer cells $^{25}$. It has been reported that miR-34a regulates glucose metabolism and tumor growth in breast cancer cells ${ }^{35}$; Overexpression of miR-122 in HCC cells can inhibit the expression of PKM2 to promote apoptosis and inhibit the migration and invasion of Hep3B cells ${ }^{36}$. In addition, IncRNA HOTAIR knockdown inhibits glycolysis by regulating miR-130a-3p/HIF1A in hepatocellular carcinoma under hypoxia ${ }^{23}$. It indicates that miR-130a-3p may be involved in the glucose metabolism process of HCC. Our study confirmed that the cell viability and colony formation rate were decreased by the miR-130a-3p mimic, while the miR-130a-3p inhibitors got the opposite results. Similarly, cell migration rate and the number of cell migration got the same result as above. Our results showed that miR-130a-3p is closely related to the progression of $\mathrm{HCC}$, which was consistent with previous reports. We have reason to believe that interfering with the expression of miR-130a-3p inhibits the glycolysis of HCC cells, reduce the production of ATP and metabolic intermediates in HCC cells, and then inhibit the proliferation and migration of HCC cells.

MicroRNAs control gene expression by binding to the 3'UTR of the target genes and inhibiting translation or inducing degradation of the target gene ${ }^{37}$. Our results showed that miR-130a-3p had no influence on the mRNA expression of most glucose metabolism related genes save for PDK1, which was the enzyme gene with maximum degree of variation of expression level, suggesting that PDK1 may be a key molecule in the regulation of glucose metabolism pathway mediated by miR-130a-3p in HCC. The bioinformatics

Page 12/22 
website was used to predict the binding site between the related glucose metabolism enzyme genes 3'UTR and miR-130a-3p, in which only PDK1 and miR-130a-3p had theoretical binding sites, and double luciferase reporter gene experiment confirmed this conclusion. In addition, overexpression of miR-130a-3p also inhibited the expression of PDK1 protein. It is reported that PDK1 is highly expressed in a variety of cancers, including liver cancer, which is consistent with our conclusion. We also found that the high expression of PDK1 is related to the lower survival rate of patients. Using the database, we concluded that the expression level of miR-130a-3p in clinical HCC was negatively correlated with the expression level of PDK1. Our study confirmed that PDK1 is a direct target of HCC and is negatively regulated by miR-130a-3p. The rescue experiment also showed that the effect of miR-130a-3p on the metabolism and progression of HCC depended on PDK1. Although we found for the first time that miR-130a-3p regulates HCC glycolysis and progress through PDK1, which enriched the theoretical system of miR-130a-3p in $\mathrm{HCC}$, the role of miR-130a-3p in vivo still needs further research.

In conclusion, our findings provide strong evidence that the down-regulation of miR-130a-3p may be the key mechanism of the up-regulation of PDK1 expression in HCC Our biological function study shows that miR-130a-3p mainly damages the proliferation and migration of $\mathrm{HCC}$ by targeting PDK1. What's more, miR-130a-3p affects the glycolysis of HCC cells by directly targeting PDK1, leading the cells more inclined to aerobic oxidation. These results manifest that the novel miR-130a-3p/PDK1 axis is crucial in reprogramming HCC glucose metabolism and regulates the proliferation and migration of HCC cells. To do our bit, our results may provide a potentially diagnostic marker and therapeutic target for the treatment of HCC.

\section{Declarations}

\section{Conflict of interest}

The authors declare that they have no competing interests, and all authors confirm its accuracy.

\section{Availability of data and materials}

The data supporting the results of this study is not publicly available, and you can obtain it from the corresponding author upon reasonable request.

\section{References}

1. Zhu, W. et al. Identification of a-fetoprotein-specific T-cell receptors for hepatocellular carcinoma immunotherapy. Hepatology 68, 574-589, doi:10.1002/hep.29844 (2018).

2. Global Burden of Disease Cancer, C. et al. Global, Regional, and National Cancer Incidence, Mortality, Years of Life Lost, Years Lived With Disability, and Disability-Adjusted Life-years for 32 Cancer 
Groups, 1990 to 2015: A Systematic Analysis for the Global Burden of Disease Study. JAMA Onco/3, 524-548, doi:10.1001/jamaoncol.2016.5688 (2017).

3. Welzel, T. M. et al. Population-attributable fractions of risk factors for hepatocellular carcinoma in the United States. Am J Gastroenterol 108, 1314-1321, doi:10.1038/ajg.2013.160 (2013).

4. Kao, W. Y. et al. A comparison of prognosis between patients with hepatitis B and C virus-related hepatocellular carcinoma undergoing resection surgery. World J Surg 35, 858-867, doi:10.1007/s00268-010-0928-z (2011).

5. Torre, L. A. et al. Global cancer statistics, 2012. CA Cancer J Clin 65, 87-108, doi:10.3322/caac.21262 (2015).

6. Esteller, M. Non-coding RNAs in human disease. Nat Rev Genet 12, 861-874, doi:10.1038/nrg3074 (2011).

7. Asano, N. et al. A serum microRNA classifier for the diagnosis of sarcomas of various histological subtypes. Nat Commun 10, 1299, doi:10.1038/s41467-019-09143-8 (2019).

8. Zeng, X. Y. et al. circCELSR1 facilitates ovarian cancer proliferation and metastasis by sponging miR598 to activate BRD4 signals. Mol Med 26, 70, doi:10.1186/s10020-020-00194-y (2020).

9. Wang, B. C. \& Ma, J. Role of MicroRNAs in Malignant Glioma. Chin Med J (Engl) 128, 1238-1244, doi:10.4103/0366-6999.156141 (2015).

10. Fu, X. T. et al. MicroRNA-30a suppresses autophagy-mediated anoikis resistance and metastasis in hepatocellular carcinoma. Cancer Lett 412, 108-117, doi:10.1016/j.canlet.2017.10.012 (2018).

11. Fu, X. et al. miR-26a enhances miRNA biogenesis by targeting Lin28B and Zcchc11 to suppress tumor growth and metastasis. Oncogene 33, 4296-4306, doi:10.1038/onc.2013.385 (2014).

12. Deng, B. et al. MiRNA-203 suppresses cell proliferation, migration and invasion in colorectal cancer via targeting of EIF5A2. Sci Rep 6, 28301, doi:10.1038/srep28301 (2016).

13. Chen, Y. et al. MicroRNA-21 down-regulates the expression of tumor suppressor PDCD4 in human glioblastoma cell T98G. Cancer Lett 272, 197-205, doi:10.1016/j.canlet.2008.06.034 (2008).

14. Weeraratne, S. D. et al. miR-34a confers chemosensitivity through modulation of MAGE-A and p53 in medulloblastoma. Neuro Onco/13, 165-175, doi:10.1093/neuonc/noq179 (2011).

15. Cui, J. G. et al. Micro-RNA-128 (miRNA-128) down-regulation in glioblastoma targets ARP5 (ANGPTL6), Bmi-1 and E2F-3a, key regulators of brain cell proliferation. J Neuroonco/ 98, 297-304, doi:10.1007/s11060-009-0077-0 (2010).

16. Kefas, B. et al. microRNA-7 inhibits the epidermal growth factor receptor and the Akt pathway and is down-regulated in glioblastoma. Cancer Res 68, 3566-3572, doi:10.1158/0008-5472.CAN-07-6639 (2008).

17. Wang, M. et al. Effect of exosome biomarkers for diagnosis and prognosis of breast cancer patients. Clin Transl Oncol 20, 906-911, doi:10.1007/s12094-017-1805-0 (2018).

18. Yu, X. F. et al. The role of miR-130a-3p and SPOCK1 in tobacco exposed bronchial epithelial BEAS-2B transformed cells: Comparison to A549 and H1299 lung cancer cell lines. $J$ Toxicol Environ Health A 
82, 862-869, doi:10.1080/15287394.2019.1664479 (2019).

19. Ouyang, M. et al. MicroRNA profiling implies new markers of chemoresistance of triple-negative breast cancer. PLoS One 9, e96228, doi:10.1371/journal.pone.0096228 (2014).

20. Kong, X., Zhang, J., Li, J., Shao, J. \& Fang, L. MiR-130a-3p inhibits migration and invasion by regulating RAB5B in human breast cancer stem cell-like cells. Biochem Biophys Res Commun 501, 486-493, doi:10.1016/j.bbrc.2018.05.018 (2018).

21. Chen, X. et al. MiR-130a-3p inhibits the viability, proliferation, invasion, and cell cycle, and promotes apoptosis of nasopharyngeal carcinoma cells by suppressing BACH2 expression. Biosci Rep 37, doi:10.1042/BSR20160576 (2017).

22. Liu, Y. et al. MiR-130a-3p regulates cell migration and invasion via inhibition of Smad4 in gemcitabine resistant hepatoma cells. J Exp Clin Cancer Res 35, 19, doi:10.1186/s13046-016-0296-0 (2016).

23. Mingxing Hu, Q. F., Chan Jing, Xu Zhang, Tao Qin,Yanfeng Pand,. LncRNA HOTAIR knockdown inhibits glycolysis by regulating miR-130a-3p/HIF1A in hepatocellular carcinoma under hypoxia. Biomedicine \& Pharmacotherapy, doi:10.1016/j.biopha.2019.109703 (2019).

24. Dafni, H. et al. Hyperpolarized $13 \mathrm{C}$ spectroscopic imaging informs on hypoxia-inducible factor-1 and myc activity downstream of platelet-derived growth factor receptor. Cancer Res 70, 7400-7410, doi:10.1158/0008-5472.CAN-10-0883 (2010).

25. Vander Heiden, M. G., Cantley, L. C. \& Thompson, C. B. Understanding the Warburg effect: the metabolic requirements of cell proliferation. Science 324, 1029-1033, doi:10.1126/science.1160809 (2009).

26. Nana-Sinkam, S. \& Croce, C. J. G. b. MicroRNA regulation of tumorigenesis, cancer progression and interpatient heterogeneity: towards clinical use. Genome Biol 15, 445, doi:10.1186/s13059-014-04458 (2014).

27. Poteet, E. et al. Reversing the Warburg effect as a treatment for glioblastoma. J Biol Chem 288, 91539164, doi:10.1074/jbc.M112.440354 (2013).

28. Kim, J. W., Tchernyshyov, I., Semenza, G. L. \& Dang, C. V. HIF-1-mediated expression of pyruvate dehydrogenase kinase: a metabolic switch required for cellular adaptation to hypoxia. Cell Metab $\mathbf{3}$, 177-185, doi:10.1016/j.cmet.2006.02.002 (2006).

29. Fujiwara, S. et al. PDK1 inhibition is a novel therapeutic target in multiple myeloma. Br J Cancer 108, 170-178, doi:10.1038/bjc.2012.527 (2013).

30. Shen, Y. C. et al. Activating oxidative phosphorylation by a pyruvate dehydrogenase kinase inhibitor overcomes sorafenib resistance of hepatocellular carcinoma. Br J Cancer 108, 72-81, doi:10.1038/bjc.2012.559 (2013).

31. Jha, M., Suk, K. J. B. t. r. \& treatment. Pyruvate dehydrogenase kinase as a potential therapeutic target for malignant gliomas. Brain Tumor Res Treat 1, 57-63, doi:10.14791/btrt.2013.1.2.57 (2013).

32. Wen, L. Z. et al. SHP-1 Acts as a Tumor Suppressor in Hepatocarcinogenesis and HCC Progression. Cancer Res 78, 4680-4691, doi:10.1158/0008-5472.CAN-17-3896 (2018). 
33. Shang, R. Z., Qu, S. B. \& Wang, D. S. Reprogramming of glucose metabolism in hepatocellular carcinoma: Progress and prospects. World J Gastroenterol 22, 9933-9943, doi:10.3748/wjg.v22.i45.9933 (2016).

34. Xu, S. \& Herschman, H. R. A Tumor Agnostic Therapeutic Strategy for Hexokinase 1-Null/Hexokinase 2-Positive Cancers. Cancer Res 79, 5907-5914, doi:10.1158/0008-5472.Can-19-1789 (2019).

35. Xiao, X. et al. The miR-34a-LDHA axis regulates glucose metabolism and tumor growth in breast cancer. Sci Rep 6, 21735, doi:10.1038/srep21735 (2016).

36. Xu, Q. et al. MicroRNA-122 affects cell aggressiveness and apoptosis by targeting PKM2 in human hepatocellular carcinoma. Oncol Rep 34, 2054-2064, doi:10.3892/or.2015.4175 (2015).

37. Iwakawa, H. O. \& Tomari, Y. The Functions of MicroRNAs: mRNA Decay and Translational Repression. Trends Cell Bio/ 25, 651-665, doi:10.1016/j.tcb.2015.07.011 (2015).

\section{Figures}



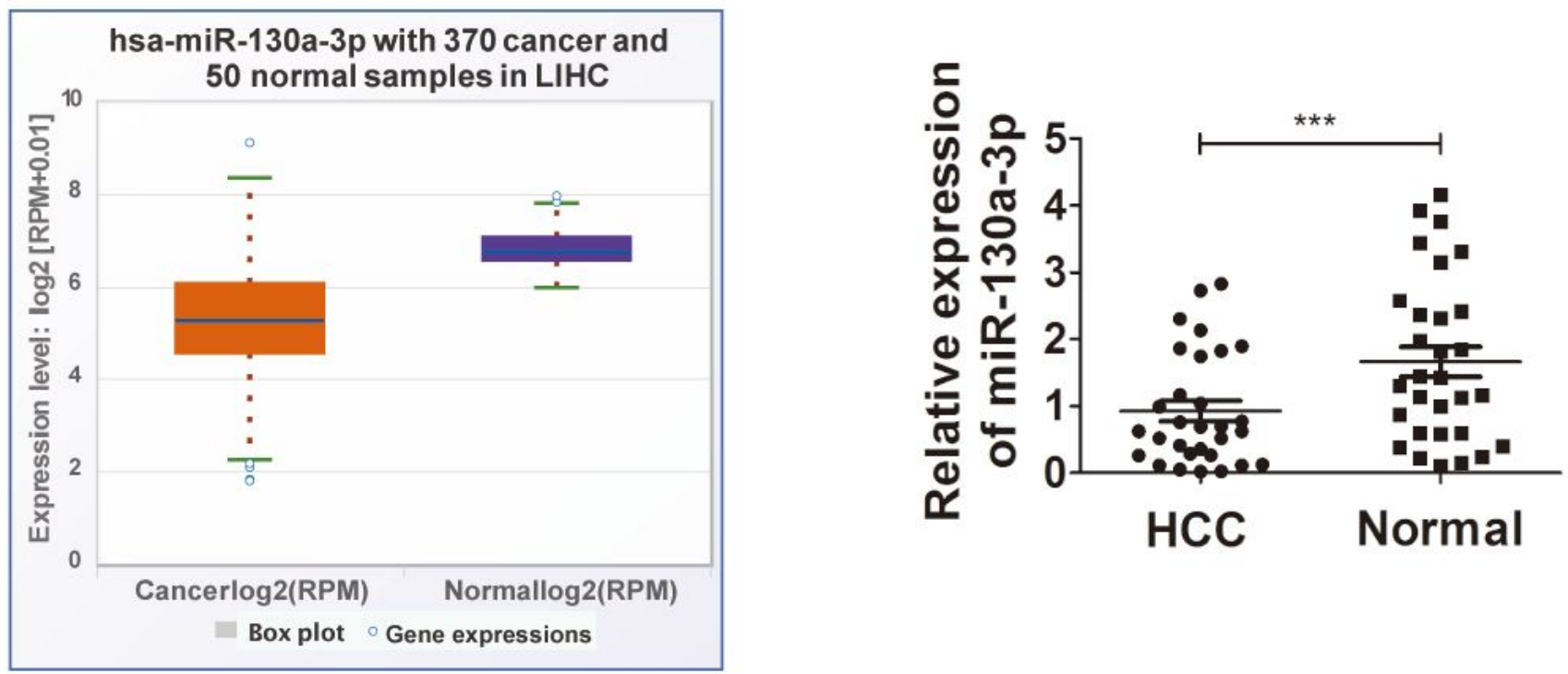

(c)

(d)

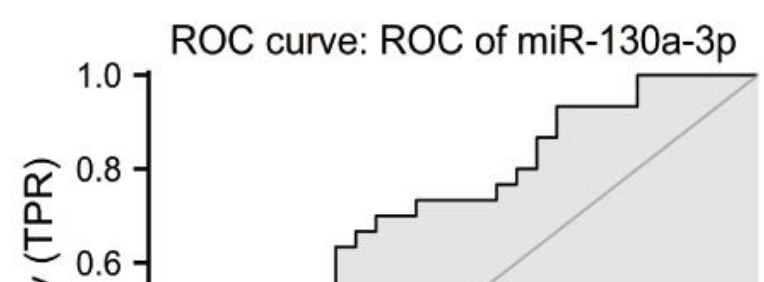

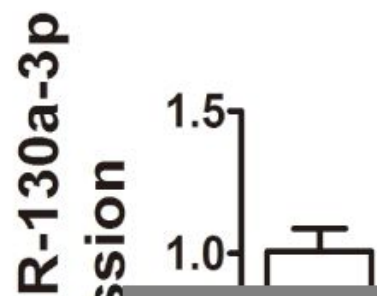

Figure 1

MiR-130a-3p was low expressed in HCC tissues and cell lines. (a) ENCORI was utilized to predict the expression of miR-130a-3p in HCC and adjacent tissues. (b) The expression of miR-130a-3p was measured by qRT-PCR in 30 cases of HCC and adjacent tissue chips. (c) The ROC curve of miR-130a-3p in HCC and adjacent tissues. (d) The expression of miR-130a-3p was measured by qRT-PCR in HCC cells. ${ }^{*} p<0.05,{ }^{* \star} p<0.01$ and ${ }^{* \star *} p<0.001$ at Student's $t$ test for indicated points relative to their control experiments. 
(a)

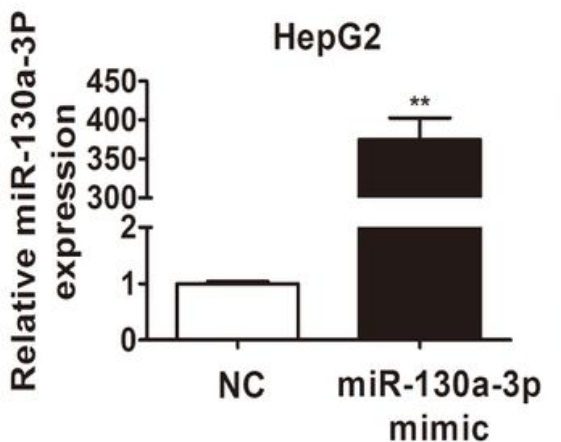

(c)

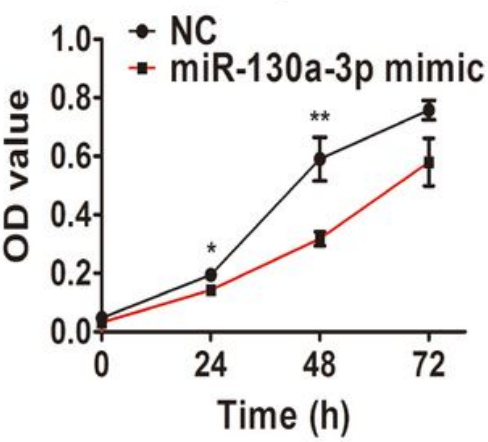

(d)

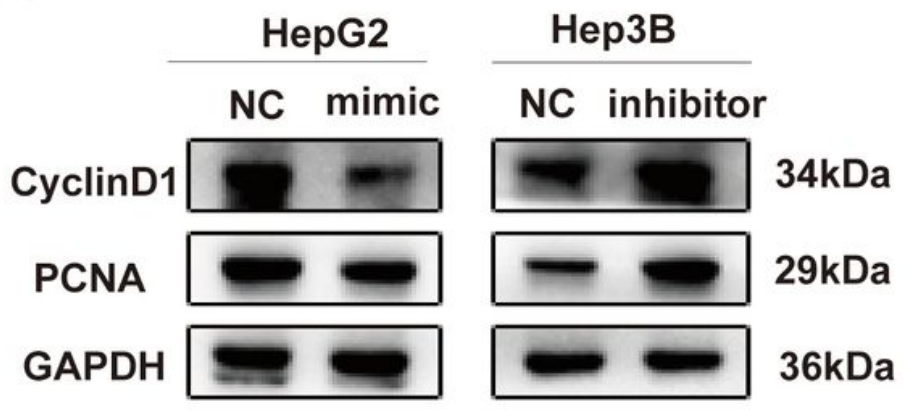

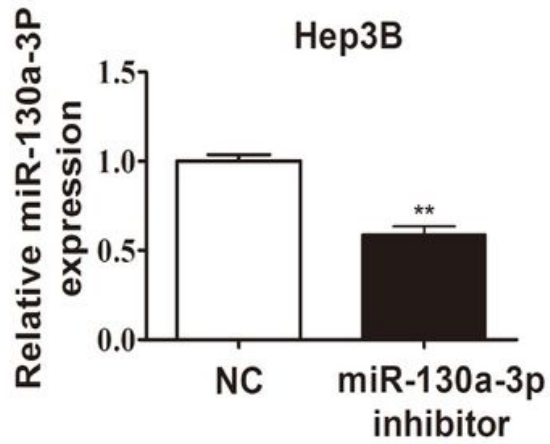

(b)

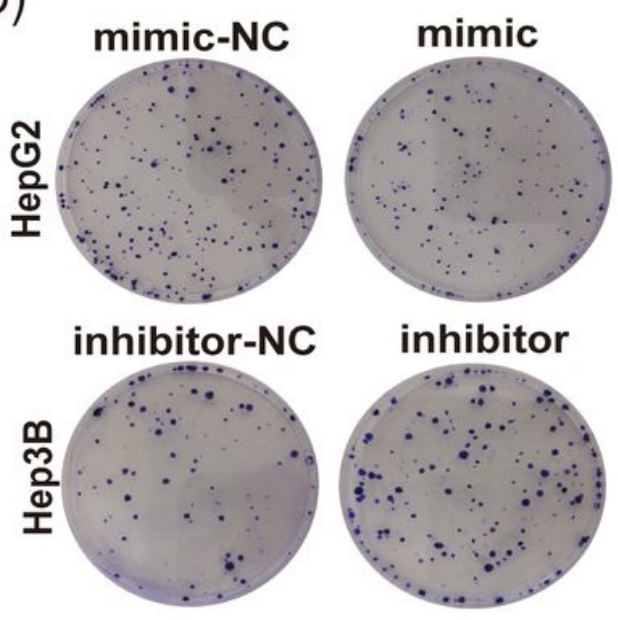

Hep3B
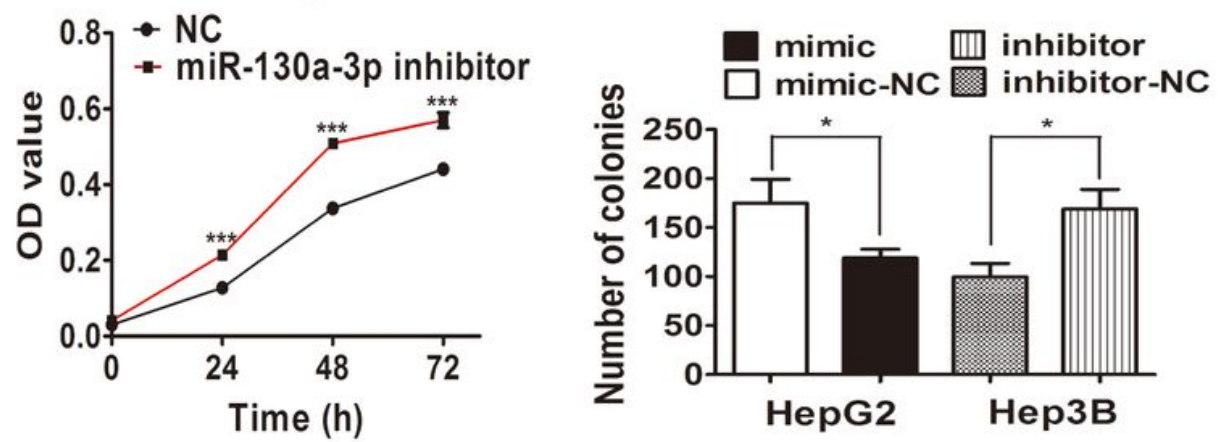

Figure 2

Overexpression of miR-130a-3p inhibits the proliferation of HCC cells. (a) MiR-130a-3p expression was detected by qRT-PCR in HepG2 cells transfected with miR-130a-3p mimic, mimic-NC or in Hep3B cells transfected with miR-130a-3p inhibitor, inhibitor-NC. (b, c) The cell proliferation was detected by CCK-8 and Clone formation in HepG2 cells transfected with miR-130a-3p mimic, mimic-NC or in Hep3B cells transfected with miR-130a-3p inhibitor, inhibitor-NC. (d) The expression of CyclinD1 and PCNA was measured by Western blot in HepG2 cells transfected with miR-130a-3p mimic, mimic-NC or in Hep3B cells transfected with miR-130a-3p inhibitor, inhibitor-NC. GAPDH was served as internal control. * $p<0.05$, ${ }^{\star *} p<0.01$ and ${ }^{* \star \star} p<0.001$ at Student's $t$ test for indicated points relative to their control experiments. 
(a)
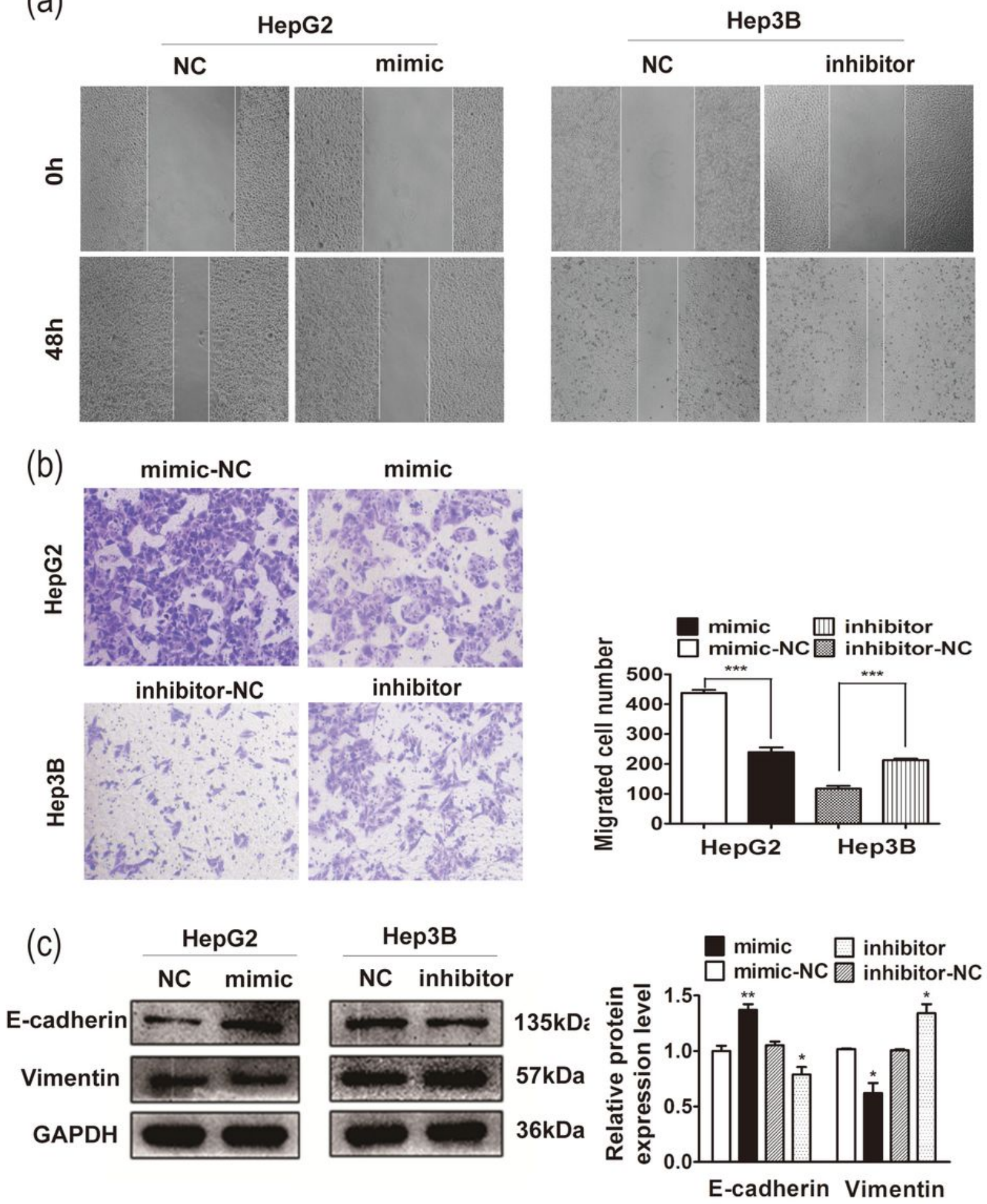

Figure 3

Overexpression of miR-130a-3p inhibits the migration of HCC cells. $(a, b)$ The cell migration was detected by Transwell and Cell scratch assay in HepG2 cells transfected with miR-130a-3p mimic, mimic-NC or in Hep3B cells transfected with miR-130a-3p inhibitor, inhibitor-NC. (c) The expression of E-cadherin and Vimentin was measured by Western blot in HepG2 cells transfected with miR-130a-3p mimic, mimic-NC or in Hep3B cells transfected with miR-130a-3p inhibitor, inhibitor-NC. GAPDH was served as internal control. 
${ }^{*} p<0.05,{ }^{*} p<0.01$ and ${ }^{* *} p<0.001$ at Student's $t$ test for indicated points relative to their control experiments.

(a)

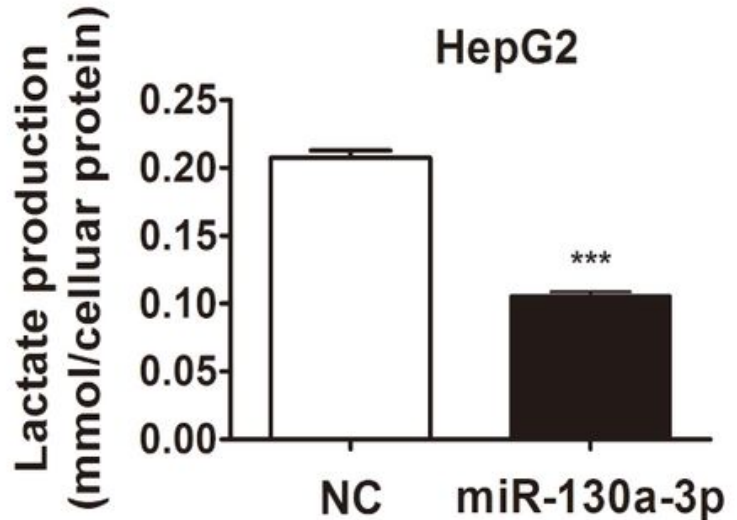

(b)

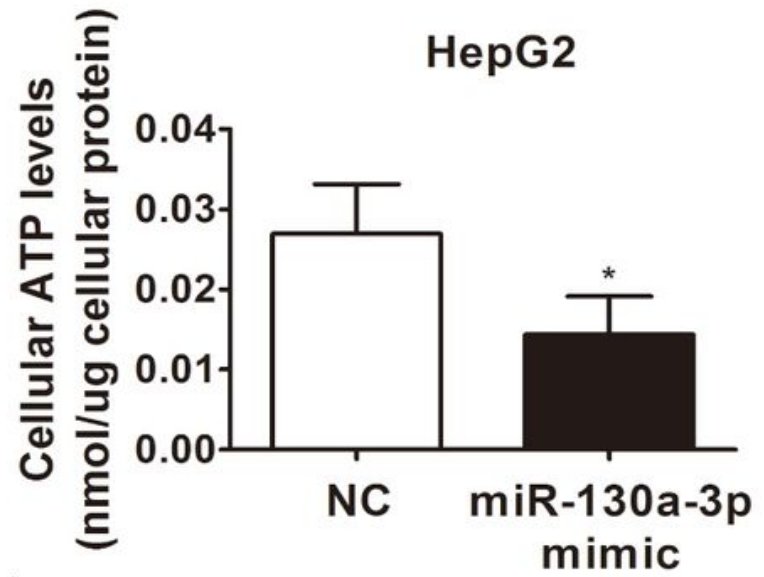

(c)

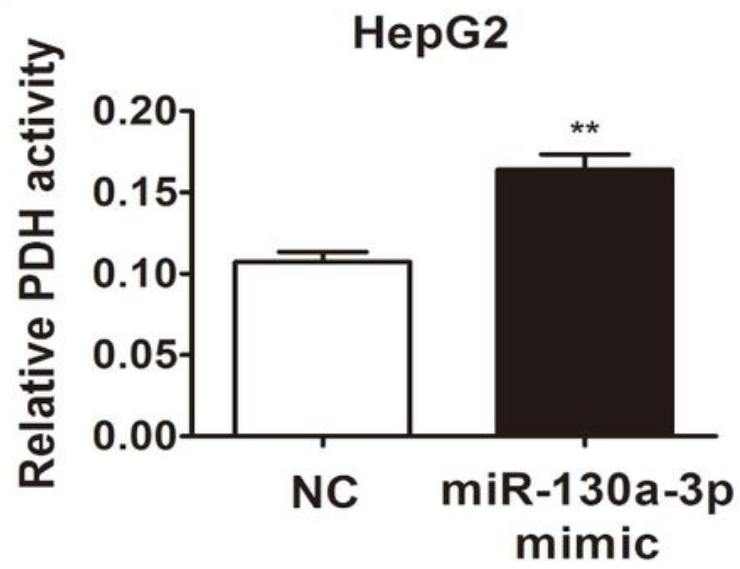

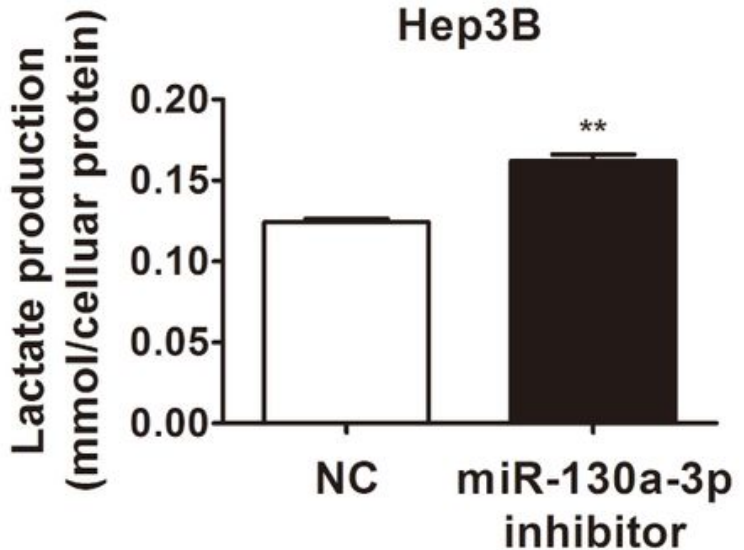

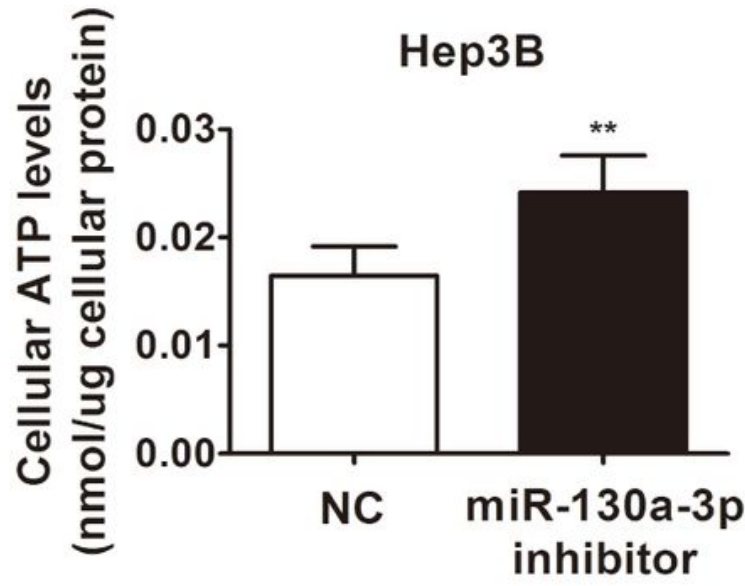

Hep3B

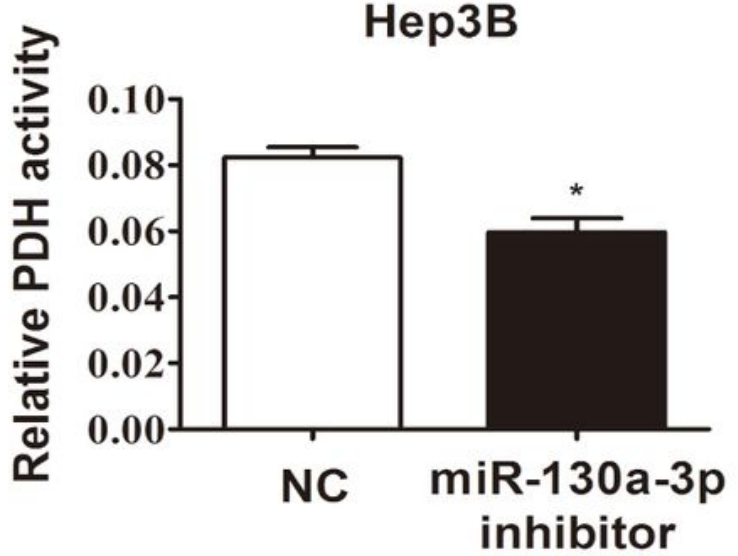

Figure 4

The effect of miR-130a-3p on glucose metabolism in HCC cells. (a) The lactate production was detected by lactic acid assay kit in HepG2 cells transfected with miR-130a-3p mimic, mimic-NC or in Hep3B cells 
transfected with miR-130a-3p inhibitor, inhibitor-NC. (b) The ATP production was detected by ATP assay kit in HepG2 cells transfected with miR-130a-3p mimic, mimic-NC or in Hep3B cells transfected with miR130a-3p inhibitor, inhibitor-NC. (c) The PDH activity was detected by PDH activity assay kit in HepG2 cells transfected with miR-130a-3p mimic, mimic-NC or in Hep3B cells transfected with miR-130a-3p inhibitor, inhibitor-NC. ${ }^{*} p<0.05,{ }^{*} p<0.01$ and ${ }^{* \star \star} p<0.001$ at Student's $t$ test for indicated points relative to their control experiments.

\section{Figure 5}

Selecting and identifying gene targeted and regulated by miR-130a-3p. (a) The expression of genes related to glucose metabolism was detected by qRT-PCR in HepG2 cells transfected with miR-130a-3p mimic, mimic-NC. (b) Using ENCORI database to analyze the correlation between miR-130a-3p and PDK1 expression in HCC tissues. (c) The expression of PDK1 was measured by Western blot in HepG2 cells transfected with miR-130a-3p mimic, mimic-NC or in Hep3B cells transfected with miR-130a-3p inhibitor, inhibitor-NC. GAPDH was served as internal control. (d) Using Schematic diagram showing the miR-130a$3 p$ targeted PDK1 sequence and the mutant binding site sequence inserted into psicheck-2. (e) The fluorescence intensity was detected by dual luciferase reporter gene detection kit in 293T cells cotransfected with miR-130a-3p mimic or mimic-NC and psicheck2-PDK1-3'UTR (WT/MUT). * $p<0.05$, ${ }^{* *} p<0.01$ and ${ }^{* * *} p<0.001$ at Student's $t$ test for indicated points relative to their control experiments.

(a)

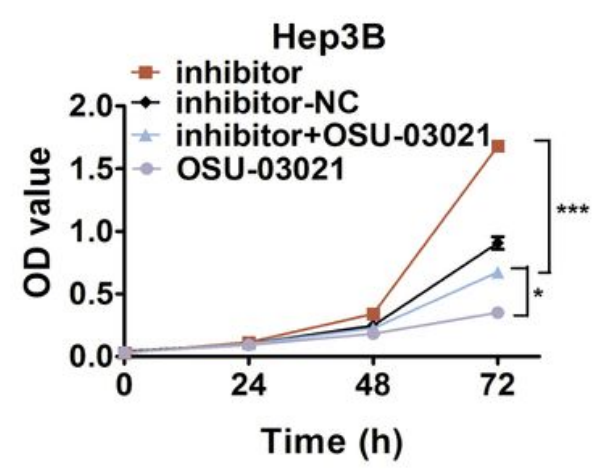

(d)

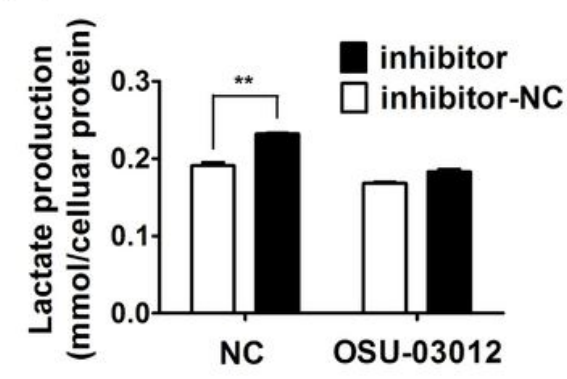

(b)

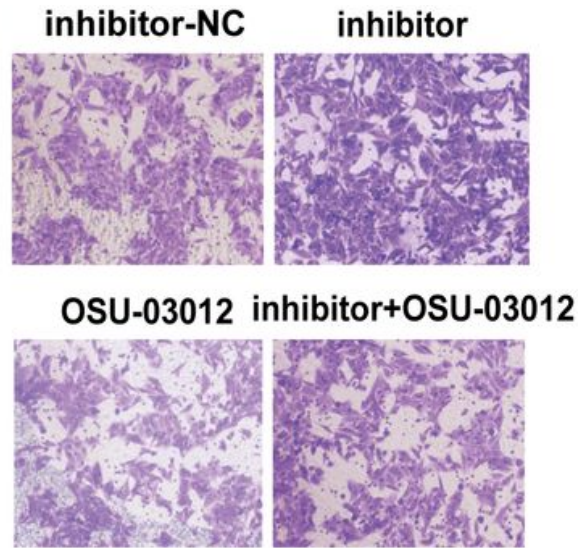

(e)

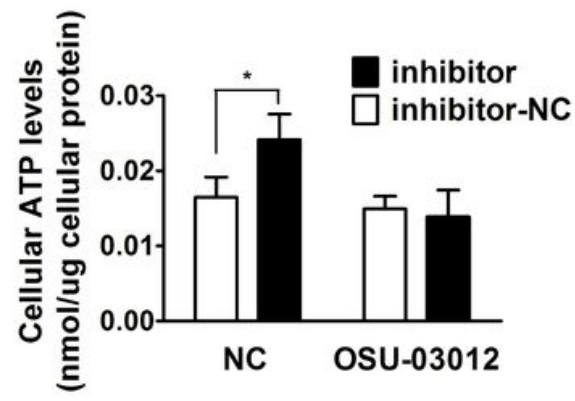

(c)

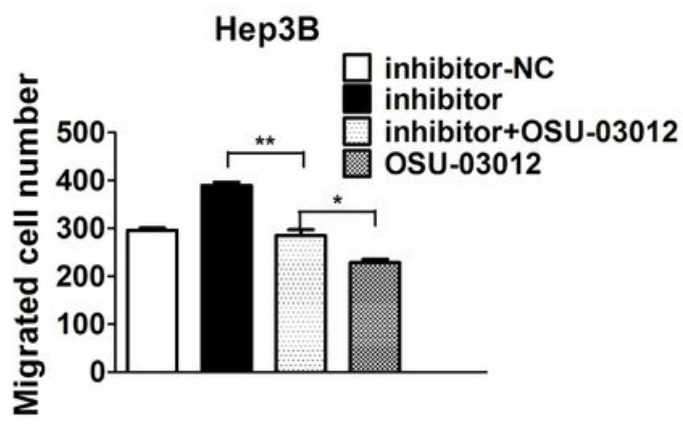

(f)

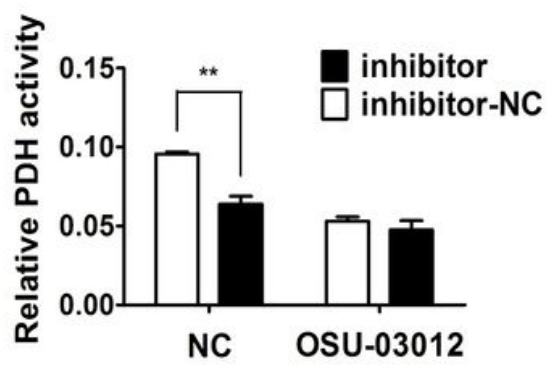




\section{Figure 6}

The effect of miR-130a-3p regulates PDK1 on the proliferation, migration and glucose metabolism of HCC cells. (a) Cell proliferation was detected by CCK-8 in 0SU-03012-treated Hep3B cells transfected with miR-130a-3p inhibitor or inhibitor-NC. (b) Cell migration was detected by cell scratch assay in 0SU-03012treated Hep3B cells transfected with miR-130a-3p inhibitor or inhibitor-NC. (c) The lactate production was detected by lactic acid assay kit in OSU-03012-treated Hep3B cells transfected with miR-130a-3p inhibitor or inhibitor-NC. (d) The ATP production was detected by ATP assay kit in OSU-03012-treated Hep3B cells transfected with miR-130a-3p inhibitor or inhibitor-NC. (e) The PDH activity was detected by PDH activity assay kit in OSU-03012-treated Hep3B cells transfected with miR-130a-3p inhibitor or inhibitor-NC. ${ }^{*} p<0.05,{ }^{*} p<0.01$ and ${ }^{* *} p<0.001$ at student's $t$ test for indicated points relative to their control experiments. 